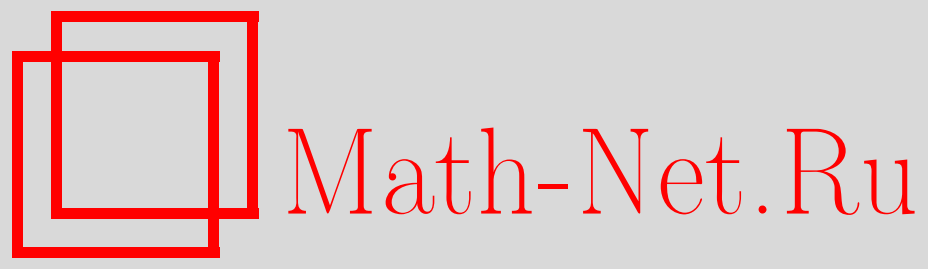

Г. С. Мустафаев, О радикале алгебр, порожденных сжатием, Функи. анализ и его прил., 2010, том 44, выпуск 3, 80-84

DOI: https://doi.org/10.4213/faa2998

Использование Общероссийского математического портала Math$\mathrm{Net.Ru} \mathrm{подразумевает,} \mathrm{что} \mathrm{вы} \mathrm{прочитали} \mathrm{и} \mathrm{согласны} \mathrm{с} \mathrm{пользователь-}$ ским соглашением

http://www.mathnet.ru/rus/agreement

Параметры загрузки:

IP : 54.172 .240 .79

26 апреля 2023 г., 16:11:14

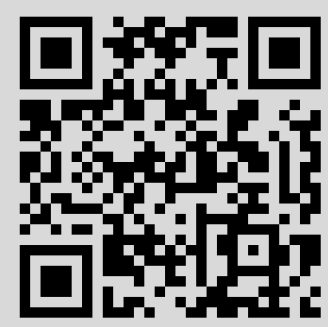


УДК 517.98

\title{
О радикале алгебр, порожденных сжатием
}

\author{
(c) 2010. Г. С. МустаФАЕВ
}

Пусть $X$ - комплексное банахово пространство и $B(X)$ - алгебра ограниченных линейных операторов в $X$. Через $\sigma(T)$ будем обозначать спектр, а через $R(z, T):=(z I-T)^{-1}$ - резольвенту оператора $T \in B(X)$. Открытый единичный круг мы будем обозначать через $D$, а единичную окружность комплексной плоскости - через Г. Если $A$ - комплексная коммутативная банахова алгебра, то через $\operatorname{Rad}(A)$ будем обозначать ее радикал. Если $\operatorname{Rad}(A)=\{0\}$, то алгебра $A$ называется полупростой. Хорошо известно, что если $A$ - коммутативная подалгебра алгебры $B(X)$, то $\operatorname{Rad}(A)=\{R \in A: \sigma(R)=\{0\}\}$.

Если $T \in B(X)$, то через $A_{T}$ (соответственно $W_{T}$ ) будем обозначать замыкание в равномерной (соответственно в слабой) операторной топологии множества полиномов от $T$. Заметим, что $A_{T}$ и $W_{T}$ - коммутативные банаховы алгебры с единицей. Напомним, что $V \in B(X)$ называется изометрией, если $\|V x\|=\|x\|$ для всех $x \in X$. Хорошо известно, что если $V-$ изометрия, то либо $\sigma(V)=\bar{D}$, либо $\sigma(V) \subset \Gamma$. Теорема Фельдмана [1] утверждает, что если $V$ - изометрия со счетным спектром, то $A_{V}$ - полупростая алгебра. Горин [2] получил более общий результат в контексте банаховых алгебр. В этой заметке мы изучаем асимптотическое поведение радикала в алгебрах, порожденных сжатием.

Напомним, что $T \in B(X)$ называется сжсатием, если $\|T\| \leqslant 1$. Если $T-$ сжатие, то $\sigma(T) \subset \bar{D}$. Множество $\sigma_{u}(T):=\sigma(T) \cap \Gamma$ называется унитарным спектром оператора $T$. Функционал $\varphi \in X^{*}$ называется унимодулярным собственным вектором оператора $T^{*}$, если $T^{*} \varphi=\xi \varphi, \xi \in \Gamma$. Следующая теорема является дискретной версией теоремы Арендта-Бетти-Любича-Ву [3, теорема $5.5 .7]$.

Теорема 1. Пусть $T-$ сжатие в банаховом пространстве $X$ со счетным унитарным спектром и $N-$ слабо* замкнутая линейная оболочка в $X^{*}$ множества унимодулярных собственных векторов оператора $T^{*}$. Тогда при всех $x \in X$

$$
\lim _{n \rightarrow \infty}\left\|T^{n} x\right\|=\sup \{|\psi(x)|: \psi \in N,\|\psi\| \leqslant 1\} .
$$

Хорошо известно, что если $T \in B(X)$, то структурное пространство $M_{T}$ алгебры $A_{T}$ можно отождествить с $\sigma_{A_{T}}(T)$ - спектром оператора $T$ относительно алгебры $A_{T}$. Так как $\sigma(T)$ является (замкнутым) подмножеством в $\sigma_{A_{T}}(T)$, то отсюда следует, что для произвольного $\xi \in \sigma(T)$ существует такой мультипликативный функционал $\phi_{\xi}$ на $A_{T}$, что $\phi_{\xi}(T)=\xi$. Применяя теорему 1 , получаем следующее обобщение теоремы Фельдмана.

Теорема 2. Пусть $T$ - сэсатие в банаховом пространстве со счетным унитарным спектром и $M-$ слабо* замкнутая линейная оболочка множества $\left\{\phi_{\xi}: \xi \in \sigma_{u}(T)\right\}$ в $A_{T}^{*}$. Тогда при всех $R \in A_{T}$

$$
\lim _{n \rightarrow \infty}\left\|T^{n} R\right\|=\sup \{|\psi(R)|: \psi \in M,\|\psi\| \leqslant 1\} .
$$

Доказательство. Пусть $L_{T}$ - оператор левого умножения на $T$ в $A_{T}$, $L_{T} S=T S, S \in A_{T}$. Очевидно, что $L_{T}$ является сжатием. Как известно [4, 
c. 232], $\sigma\left(L_{T}\right)$ совпадает с объединением спектра $\sigma(T)$ и ограниченных компонент резольвентного множество оператора $T$. Отсюда следует, что $\sigma_{u}\left(L_{T}\right)=$ $\sigma_{u}(T)$. С другой стороны, легко проверить, что множество собственных векторов оператора $L_{T}^{*}$ совпадает с множеством $\left\{\lambda \phi: \phi \in M_{T}, \lambda \in \mathbb{C}\right\}$. Кроме того, из теоремы Шилова [5, теорема 2.3.1] следует, что $\sigma_{A_{T}}(T) \cap \Gamma=\sigma_{u}(T)$. Следовательно, множество унимодулярных собственных векторов оператора $L_{T}^{*}$ совпадает с множеством

$$
\left\{\lambda \phi_{\xi}: \xi \in \sigma_{u}(T), \lambda \in \mathbb{C}\right\} .
$$

Теперь, применяя теорему 1 к оператору $L_{T}$ в пространстве $A_{T}$, получим требуемое утверждение.

Если спектр оператора $T \in B(X)$ содержится в $\Gamma$, то структурное пространство алгебры $A_{T}$ можно отождествить с $\sigma(T)$ [5, следствие 2.3.2]. Значит, справедливо

Следствие 3. Если T- сжатие в банаховом пространстве со счетным унитарным спектром, то справедливы следующие утверждения:

(a) $\lim _{n \rightarrow \infty}\left\|T^{n} R\right\|=0$ для каждого $R \in \operatorname{Rad}\left(A_{T}\right)$;

(b) если $\sigma(T) \subset \Gamma$, mo $\operatorname{Rad}\left(A_{T}\right)=\left\{R \in A_{T}: \lim _{n \rightarrow \infty}\left\|T^{n} R\right\|=0\right\}$.

Другое обобщение теоремы Фельдмана:

Теорема 4. Если $T$ - сжатие в банаховом пространстве $X$ со счетным унитарным спектром, то для произвольных $R \in \operatorname{Rad}\left(W_{T}\right)$ u $x \in X$

$$
\lim _{n \rightarrow \infty}\left\|T^{n} R x\right\|=0 .
$$

Доказательство. Пусть $\varphi \in X^{*}$ является унимодулярным собственным вектором оператора $T^{*}$, соответствующим собственному значению $\xi \in \Gamma$, и пусть $R \in \operatorname{Rad}\left(W_{T}\right)$. В силу теоремы 1 достаточно показать, что $R^{*} \varphi=0$. Так как $R \in W_{T}$, то существует направленность полиномов $\left\{P_{i}\right\}_{i \in I}$, такая, что $P_{i}(T) \rightarrow R$ в слабой операторной топологии. Пусть $x \in \operatorname{Ker} \varphi$. Так как $P_{i}(T)^{*}=P_{i}(\xi) \varphi$, то

$$
0=P_{i}(\xi)\langle\varphi, x\rangle=\left\langle P_{i}(T)^{*} \varphi, x\right\rangle \rightarrow\left\langle R^{*} \varphi, x\right\rangle .
$$

Это показывает, что $\operatorname{Ker} \varphi \subset \operatorname{Ker} R^{*} \varphi$ и потому $R^{*} \varphi=\lambda \varphi$ для некоторого $\lambda \in \mathbb{C}$. Так как $R \in \operatorname{Rad}\left(W_{T}\right)$, получаем, что $R^{*} \varphi=0$.

Для произвольных $T \in B(X)$ и $x \in X$ обозначим через $\rho_{T}(x)$ множество всех тех точек $\lambda \in \mathbb{C}$, для которых существует $X$-значная функция $u(z)$, аналитическая в некоторой окрестности $U_{\lambda}$ точки $\lambda$ и такая, что $(z I-T) u(z)=x$ при всех $z \in U_{\lambda}$. Это множество является открытым и содержит резольвентное множество $\rho(T)$ оператора $T$. Локальный спектр $\sigma_{T}(x)$ оператора $T$ в точке $x$ определяется как дополнение к $\rho_{T}(x)$ и потому является замкнутым подмножеством в $\sigma(T)$. Множество $\sigma_{T}(x) \cap \Gamma$ называется локальным унитарным спектром оператора $T$ в точке $x$.

Ниже мы докажем локальный вариант теоремы 4.

Теорема 5. Пусть $T$ - сжатие в банаховом пространстве $X$ u $x \in X$. Если $\sigma_{T}(x) \cap \Gamma-$ счетное множество, то для произвольного $R \in \operatorname{Rad}\left(W_{T}\right)$

$$
\lim _{n \rightarrow \infty}\left\|T^{n} R x\right\|=0 .
$$

Для доказательства нам понадобятся некоторые леммы.

Лемма 6. Пусть $V$ - изометрия в банаховом пространстве $X$ u $x \in X$. Если $\sigma_{T}(x) \cap \Gamma-$ счетное множество, то $R x=0$ при всех $R \in \operatorname{Rad}\left(W_{V}\right)$. 
Доказательство. Положим $E=\overline{\operatorname{span}}\left\{T^{n} x: n \geqslant 0\right\}$. Тогда $E$ является $T$-инвариантным подпространством в $X$. Пусть $\left.V\right|_{E}$ - сужение изометрии $V$ на $E$. В силу [6, лемма 1.3] $\sigma_{u}\left(\left.V\right|_{E}\right)=\sigma_{V}(x) \cap \Gamma$. Следовательно, $\left.V\right|_{E}$ является изометрией со счетным спектром. Заметим также, что если $R \in \operatorname{Rad}\left(W_{V}\right)$, то $\left.R\right|_{E} \in \operatorname{Rad}\left(W_{\left.V\right|_{E}}\right)$. С другой стороны, из теоремы 4 следует, что алгебра $W_{\left.V\right|_{E}}$ полупроста. Таким образом, $\left.R\right|_{E}=0$ и, значит, $R x=0$.

Лемма 7. Если $T$ - сжатие в банаховом пространстве $X$ и $E-$-инвариантное относительно $T$ подпространство, то для произвольного $x \in E$

$$
\sigma_{\left.T\right|_{E}}(x) \cap \Gamma=\sigma_{T}(x) \cap \Gamma .
$$

Доказательство. Пусть $\pi: X \rightarrow X / E-$ каноническое отображение и $x \in$ $E$. Легко проверить, что $\sigma_{T}(x) \cap \Gamma \subset \sigma_{\left.T\right|_{E}}(x) \cap \Gamma$. Чтобы доказать обратное включение, рассмотрим $\xi \in \rho_{T}(x) \cap \Gamma$. Тогда существует некоторая окрестность $U_{\xi}$ точки $\xi$ и такая $X$-значная аналитическая в $U_{\xi}$ функция $u(z)$, что $(z I-$ $T) u(z)=x, z \in U_{\xi}$. Для произвольного $z \in U_{\xi} \cap\{z \in \mathbb{C}:|z|>1\}$ мы имеем $\pi u(z)=0$, так как

$$
u(z)=R(z, T) x=\sum_{n=0}^{\infty} z^{-n-1} T^{n} x \in E .
$$

По теореме единственности $\pi u(z)=0$ при всех $z \in U_{\xi}$ и потому $u(z) \in E$. Таким образом, $\left(z I-\left.T\right|_{E}\right) u(z)=0$ при всех $z \in U_{\xi}$. Это показывает, что $\xi \in$ $\rho_{\left.T\right|_{E}}(x) \cap \Gamma$.

Следующая лемма хорошо известна (см., например, [6] и [7, лемма 2.1]).

Лемма 8. Если $T$ - сжатие в банаховом пространстве $X$, то существуют банахово пространство $Y$, линейный сжимающий оператор $J: X \rightarrow Y c$ плотным образом и изометрия $V$ на $Y$, такие, что

1) $V J=J T$

2) $\|J x\|=\lim _{n \rightarrow \infty}\left\|T^{n} x\right\|$ при всех $x \in X$;

3) $\sigma(V) \subset \sigma(T)$.

Тройка $(Y, J, V)$ называется предельной изометрией, ассоциированной с оператором $T$.

Пусть $T$ - сжатие в банаховом пространстве $X$ и $(Y, J, V)$ - предельная изометрия, ассоциированная с $T$. Для произвольного $R \in W_{T}$ определим оператор $\widetilde{R}$ на $J X$ равенством $\widetilde{R} J x=J R x, x \in X$. Тогда

$$
\|\widetilde{R} J x\|=\|J R x\|=\lim _{n \rightarrow \infty}\left\|T^{n} R x\right\|=\lim _{n \rightarrow \infty}\left\|R T^{n} x\right\| \leqslant\|R\| \lim _{n \rightarrow \infty}\left\|T^{n} x\right\|=\|R\|\|J x\| .
$$

Поскольку $J$ имеет плотный образ, $\widetilde{R}$ допускает расширение на все $Y$. Будем его снова обозначать через $\widetilde{R}$. Легко проверить, что $\widetilde{R} \in W_{V}$ и отображение $R \rightarrow \widetilde{R}$ является сжимающим гомоморфизмом. Отсюда следует, что если $R \in \operatorname{Rad}\left(W_{T}\right)$, то $\widetilde{R} \in \operatorname{Rad}\left(W_{V}\right)$.

Доказательство теоремы 5. Положим $E=\overline{\operatorname{span}}\left\{T^{n} x: n \geqslant 0\right\}$ и $S=$ $\left.T\right|_{E}$. Пусть $(Y, J, V)$ - предельная изометрия, ассоциированная с $S$. Заметим, что $\sigma_{V}(J x) \subset \sigma_{S}(x)$. Действительно, если $\lambda \in \rho_{S}(x)$, то существует некоторая окрестность $U_{\lambda}$ точки $\lambda$ и такая $E$-значная аналитическая в $U_{\lambda}$ функция $u(z)$, что $(z I-S) u(z)=x, z \in U_{\lambda}$. Отсюда следует, что $(z J-J S) u(z)=J x$. Согласно лемме $8,(z I-V) J u(z)=J x, z \in U_{\lambda}$, так как $J S=V J$. Это показывает, что 
$\lambda \in \rho_{V}(J x)$. Следовательно, $\sigma_{V}(J x) \cap \Gamma \subset \sigma_{S}(x) \cap \Gamma$. Принимая во внимание лемму 7, имеем $\sigma_{V}(J x) \cap \Gamma \subset \sigma_{T}(x) \cap \Gamma$. Отсюда вытекает, что $\sigma_{V}(J x) \cap \Gamma$ - счетное множество. Теперь, если $R \in \operatorname{Rad}\left(W_{T}\right)$, то $Q:=\left.R\right|_{E} \in \operatorname{Rad}\left(W_{S}\right)$ и потому $\widetilde{Q} \in \operatorname{Rad}\left(W_{V}\right)$. Так как $\sigma_{V}(J x) \cap \Gamma$ счетно, то, используя лемму 6 , получаем, что $J Q x=\widetilde{Q} J x=0$ и потому $J R x=0$. В силу леммы 8 это означает, что $\lim _{n \rightarrow \infty}\left\|T^{n} R x\right\|=0$.

Пусть $T \in B(X)$. Как известно [8], если множество $\left\{T^{n}: n \in \mathbb{N}\right\}$ относительно компактно, то $\sigma_{u}(T)$ - конечное множество. Заметим, что при этом условии $T$ есть оператор с ограниченными степенями. Заменив норму пространства $X$ на эквивалентную, можно считать, что $T$ является сжатием. Теперь из теоремы 4 следует, что если множество $\left\{T^{n}: n \in \mathbb{N}\right\}$ относительно компактно, то $\lim _{n \rightarrow \infty}\left\|T^{n} R x\right\|=0$ при всех $R \in \operatorname{Rad}\left(W_{T}\right)$ и $x \in X$. Однако можно утверждать даже большее:

Теорема 9. Пусть $T \in B(X)$. Если множество $\left\{T^{n}: n \in \mathbb{N}\right\}$ относительно компактно в слабой топологии пространства $A_{T}$, то для произвольного $R \in$ $\operatorname{Rad}\left(W_{T}\right)$

$$
\lim _{n \rightarrow \infty}\left\|T^{n} R\right\|=0 .
$$

Доказательство. Поскольку $T$ - оператор с ограниченными степенями, $\sigma(T) \subset \bar{D}$. Сначала покажем, что $\sigma_{u}(T)-$ конечное множество. Пусть $\xi \in$ $\sigma_{u}(T)$. Достаточно показать, что $\{\xi\}$ является изолированной точкой в $\sigma(T)$. Так как множество $\left\{\bar{\xi}^{n} T^{n}: n \in \mathbb{N}\right\}$ относительно компактно в слабой топологии пространства $A_{T}$, можно считать, что $\xi=1$. Здесь мы будем пользоваться стандартной техникой эргодической теории. Положим $P_{n}(T)=\frac{1}{n}\left(T+\cdots+T^{n}\right)$. Из теоремы Крейна-Шмульяна [9, с. 471] следует, что множество $\left\{P_{n}(T): n \in\right.$ $\mathbb{N}\}$ относительно компактно в слабой топологии пространства $A_{T}$. По теореме Эберлейна-Шмульяна [9, с. 456] существует такая подпоследовательность $\left\{P_{n_{k}}(T)\right\}$ и $P \in B(X)$, что $P_{n_{k}}(T) \rightarrow P$ при $k \rightarrow \infty$ в слабой топологии пространства $A_{T}$. Можно убедиться, что $P \in A_{T}$. Далее, для произвольного $\xi \in \sigma(T)$ существует такой мультипликативный функционал $\phi_{\xi}$ на $A_{T}$, что $\phi_{\xi}(T)=\xi$. Следовательно,

$$
\phi_{\xi}(P)=\lim _{k \rightarrow \infty} \phi_{\xi}\left(P_{n_{k}}(T)\right)=\lim _{k \rightarrow \infty} P_{n_{k}}(\xi)=\lim _{k \rightarrow \infty} \frac{1}{n_{k}}\left(\xi+\cdots+\xi^{n_{k}}\right)= \begin{cases}0, & \xi \neq 1, \\ 1, & \xi=1 .\end{cases}
$$

Так как $\xi \mapsto \phi_{\xi}(P)-$ непрерывная функция на $\sigma(T)$, то $\{1\}$ является изолированной точкой в $\sigma(T)$.

Из равенств

$$
(T-I) P_{n_{k}}(T)=P_{n_{k}}(T)(T-I)=\frac{T^{n_{k}+1}-T}{n_{k}} \rightarrow 0 \quad(k \rightarrow \infty),
$$

получаем $T P=P T=P$. В частности, $P^{2}=P$. Легко видеть, что $P$ есть спектральный проектор, соответствующий точке $\{1\}$. Теперь пусть $R \in \operatorname{Rad}\left(W_{T}\right)$. Если $\sigma_{u}(T)=\varnothing$, то $\lim _{n \rightarrow \infty}\left\|T^{n}\right\|=0$. Поэтому можно считать, что $\sigma_{u}(T)=$ $\left\{\xi_{1}, \ldots, \xi_{k}\right\}$. Пусть $P_{i}-$ спектральный проектор, соответствующий точке $\left\{\xi_{i}\right\}$ $(i=1, \ldots, k)$ и $P=P_{1}+\cdots+P_{k}$. Так как $T P_{i}=P_{i} T=\xi_{i} P_{i}$, то $R P_{i}=$ $P_{i} R=0$, откуда следует, что $R P=P R=0$. Заметим также, что спектр опеpaтора $T(I-P)$ содержится в $D$ и потому $\lim _{n \rightarrow \infty}\left\|T^{n}(I-P)\right\|=0$. Так как $T^{n} R=T^{n}(I-P) R$, получаем, что $\lim _{n \rightarrow \infty}\left\|T^{n} R\right\|=0$. 
Напомним, что компактный и квазинильпотентный оператор в банаховом пространстве называется вольтерровым. Оператор $T \in B(X)$ называется (слабо) почти периодическим, если множество $\left\{T^{n} x: n \geqslant 0\right\}$ относительно (слабо) компактно для всех $x \in X$. Применяя теорему де Лю-Гликсберга [10] об отщеплении, мы получаем следующую теорему.

Теорема 10. (а) Если $T$ - почти периодический оператор в банаховом пространстве $X$, то для произвольных $R \in \operatorname{Rad}\left(W_{T}\right)$ u $x \in X$

$$
\lim _{n \rightarrow \infty}\left\|T^{n} R x\right\|=0 ;
$$

(b) Если T - слабо почти периодический оператор в банаховом пространстве, то для произвольного вольтеррова оператора $R$ из $W_{T}$

$$
\lim _{n \rightarrow \infty}\left\|T^{n} R\right\|=0 .
$$

Автор благодарит рецензента за ценные замечания и советы.

\title{
ЛитеРАТУРА
}

[1] Г. М. Фельдман, Функц. анализ и его прил., 8:2 (1974), 93-94. [2] Е. А. Горин, Функц. анализ и его прил., 8:2 (1974), 73-74. [3] J. van Neerven, The Asymptotic Behaviour of Semigroups of Linear Operators, Oper. Theory Adv. Appl., vol. 88, Birkhäuser, Basel, 1996. [4] Yu. I. Lyubich, Linear Functional Analysis, Encyclopaedia Math. Sci., vol. 19, Springer-Verlag, Berlin, 1992. [5] R. Larsen, Banach Algebras, Marcel Dekker, New York, 1973. [6] L. Kérchy, J. van Neerven, Acta Sci. Math. (Szeged), 63:3-4 (1997), 551-562. [7] V. Q. Phóng, J. Funct. Anal., 103:2 (1992), 74-84. [8] A. Swiech, Studia Math., 96 (1990), 277-282. [9] Н. Данфорд, Дж. Т. Шварц, Линейные операторы. Общая теория, ИЛ, М., 1962. [10] К. de Leeuw, I. Glicksberg, Acta Math., 105 (1961), 63-97.

Университет Йузунджу Йыл, Турция

e-mail: hsmustafayev@yahoo.com

Поступило в редакцию 15 июля 2009 г.

УДК $517.98+519.2$

\section{Инвариантные функционалы случайных матриц*}

\author{
(c) 2010. В. Ю. Протасов
}

Показатели (экспоненты) Ляпунова - показатели роста норм произведений случайных матриц - широко изучаются в литературе ([1]-[10]). В данной статье мы введем понятие инвариантного функционала («антинормы») для семейства случайных матриц и докажем его существование при определенных условиях. Из этого будут следовать асимптотически точные оценки средней скорости роста норм и спектральных радиусов матричных произведений, а также ряд новых оценок показателя Ляпунова. Для простоты ограничимся случаем независимых сомножителей, принимающих значения на конечном множестве матриц. Пусть $\eta_{k}=X_{k} \cdots X_{1}$, где все $X_{i}$ независимы и одинаково распределены на множестве

*Работа выполнена при поддержке грантов РФФИ 08-01-00208 и 10-01-00293, гранта МД2195.2008.1 и гранта НШ-3233.2008.1. 